\title{
Can compactness constrain the Gerrymander?
}

\author{
Macartan Humphreys * \\ Columbia University \\ mh2245@columbia.edu
}

October 10, 2009

\begin{abstract}
Gerrymandering - the manipulation of electoral boundaries to maximize constituency wins - is often seen as a pathology of democratic systems. A commonly cited cure is to require that electoral constituencies have a 'compact' shape. But how much of a constraint does compactness in fact place on would-be gerrymanderers? We operationalize compactness as a convexity constraint and apply a theorem of Kaneko, Kano, and Suzuki (2004) to the two party situation to show that for any population distribution a gerrymanderer can always create equal sized convex constituencies that translate a margin of $k$ voters into a margin of at least $k$ constituency wins. Thus even with a small margin a majority party can win all constituencies. Moreover there always exists some population distribution such that all divisions into equal sized convex constituencies translate a margin of $k$ voters into a margin of exactly $k$ constituencies. Thus a convexity constraint can sometimes prevent a gerrymanderer from generating any wins for a minority party.
\end{abstract}

\footnotetext{
${ }^{*}$ I thank Mikio Kano for generous advice on this work.
} 


\section{Introduction}

Gerrymandering is the art of manipulating electoral constituencies in order to maximize vote shares for a favored party. In principle the scope for gerrymandering is large and for this reasons lawmakers and courts have advanced criteria to limit it. Perhaps the three most common criteria are that electoral constituencies should possess equality of population (equinumerosity), contiguity, and compactness (Niemi, Grofman, Carlucci, and Hofeller, 1990; Polsby and Popper, 1991). The arguments for equinumerosity and contiguity are relatively simple; the arguments for compactness is more difficult. Without something like compactness anything goes: it is possible to satisfy contiguity and place any set of people together in a single constituency subject only to the size constraint (Polsby and Popper, 1991). Advocates also argue the converse however: that "a requirement of compactness would prevent effective gerrymandering" (Polsby and Popper, 1991) and that compactness acts as a "prophylactic measure that limits the possibility of legislative gerrymandering" (Stern, 1974). Thirty five US states now require that constituencies be compact (Chambers and Miller, 2009).

There is however considerable disagreement over what is meant by 'compact' (the mathematical notion of compactness - closed and bounded - is of limited application for this problem). Niemi, Grofman, Carlucci, and Hofeller (1990) list nearly two dozen compactness measures and advocate the use of multiple measures to capture different features of compactness (see also Young (1988)). Two prominent features discussed in the literature are the extent to which an area is 'elongated' and the extent to which it is irregularly shaped (Chambers and Miller, 2009). Dispersion measures focus on the former, convexity measures focus on the latter (where an area is convex if the shortest path between two points in the area lies entirely within the area) (Taylor, 1973; Chambers and Miller, 2009).

There are also criticisms of the use of compactness as a criterion for limiting gerrymandering. Vickrey (1961) notes that shape considerations alone can not rule out gerrymandering. Lowenstein and Steinberg (1985) argue that compactness constraints are biased, in the US benefiting Republicans over Democrats (but more generally harming parties whose support base is more clustered). The argument is dismissed for lack of supporting evidence by Polsby and Popper (1991) but is supported by simulations provided by Altman (1998). 
Following Taylor (1973) and Chambers and Miller (2009) we focus on convexity as a simple requirement for constituency compactness and ask what scope there is for gerrymandering given such a constraint. One might expect that convexity could impose formidable constraints since it rules out the tentacled shapes traditionally associated with gerrymandering. Moreover by most measures, compactness is maximized by convex shapes even if convexity is not an explicit part of the measures. For measures that focus on minimizing dispersion within districts, for example (see Fryer and Holden (2007) and related measures in Niemi, Grofman, Carlucci, and Hofeller (1990)), convexity is a necessary but not a sufficient condition for compactness.

An application of a theorem from computational geometry due to Kaneko, Kano, and Suzuki (2004) however yields a striking result. In general, a convexity requirement has no effect on the scope for gerrymandering for majority parties; narrow numeric margins are sufficient to ensure that a gerrymanderer can bank all constituencies and still comply with convexity. In addition we show here that convexity may have an adverse effect on the ability of minorities to gerrymander. For some population distributions the convexity constraint can prevent a minority party from gaining any seats, even if they fully control constituency designs and the margin between majority and minority is small. These results suggest that shape based constraints are unlikely to resolve the problems associated with gerrymandering; moreover "anonymous" procedures may be no better at ensuring even rough proportionality; the heart of the problem is not the manner in which constituency boundaries are established but the poverty of the information aggregated by single member districts.

\section{Results}

The setting we examine is idealized in a number of ways. We assume that all voters support one of two parties in single member constituencies; and we take these voters to have fixed locations which can be represented as points in "general position" on a convex two dimensional space. ${ }^{1}$ We assume that the number of voters is fixed at $n$, that the number of constituencies is fixed at $k$, and that $\frac{n}{k}$ is an odd integer.

We consider designs for the location of constituency boundaries

\footnotetext{
${ }^{1}$ Note other recent work on gerrymandering allows for more continuous party affiliation but removes geographical constraints (Friedman and Holden, 2008)
} 
and we say that a design is "admissible" if it partitions the space into $k$ convex areas with $\frac{n}{k}$ voters in each area. Implicitly then we examine 'wholesale' gerrymandering in which constituency design does not depend on preexisting designs.

Under these conditions, our first result follows from a theorem due to Kaneko, Kano, and Suzuki (2004).

Theorem 1. (Kaneko, Kano and Suzuki) Let $a \geq 1, g \geq 0$ and $h \geq 0$ be integers such that $g+h \geq 1$. Let $R$ be a set of $a g+(a+1) h$ red points and $B$ a set of $(a+1) g+$ ah blue points in the plane such that $R \cup B$ is in general position. Then there exists a subdivision $X_{1} \cup X_{2} \cup \cdots \cup X_{g} \cup Y_{1} \cup Y_{2} \cup \cdots \cup Y_{h}$ of the plane into $g+h$ disjoint convex polygons such that every $X_{i}(1 \leq i \leq g)$ contains exactly a red points and $a+1$ blue points and every $Y_{j}(1 \leq j \leq h)$ contains exactly $a+1$ red points and a blue points

The theorem generalizes a result due to Bespamyatnikh, Kirkpatrick, and Snoeyink (2000) that is in turn a generalization of the ham sandwich theorem in two dimensions. Proposition 1 below follows as a direct implication of the theorem.

Proposition 1. Let $m_{i},-k \leq m_{i} \leq k$, denote the margin of voters that party $i$ commands. Then for any population distribution in general position there exists an admissible design such that party $i$ commands a margin of $m_{i}$ constituencies.

Proof. Follows immediately from theorem 1, setting $g=\frac{k-m}{2}, h=$ $\frac{k+m}{2}, a=\frac{n-k}{2 k}$.

Proposition 1 provides a minimum number of constituencies that a gerrymander can guarantee a party. A $k$ vote margin is sufficient to guarantee $100 \%$ of constituencies. The proposition extends in the obvious way to parties with a margin greater than $k$ or less than $-k$.

With a margin of $-k$ votes the proposition does not provide a guarantee that a gerrymanderer can control any constituencies. Yet clearly it is sometimes possible for a gerrymanderer controlling margin $m_{i}<k-1$ to control more than $m_{i}$ constituencies. Indeed it is easy to see that a party that controls $v$ votes could control up to $\min \left(\left\lfloor\frac{2 k}{n+k} v\right\rfloor, k\right)$ constituencies. Thus for example in a polity with 6 constituencies each containing 15 voters, a party with 40 of 90 voters could in some circumstances organize boundaries so that it has 8 members in each of 5 convex constituencies, thereby translating a 
share of $44 \%$, and a margin of -10 votes into a constituency share of $83 \%$. In general is it always possible for parties to use gerrymandering to ensure that their constituency margin exceeds their vote margin (subject to the limit of $k$ constituencies)?

The next result answers this question in the negative and establishes that the gains guaranteed in Proposition 1 are in general the greatest gains that can be guaranteed by a gerrymanderer.

Proposition 2. Let $m_{i},-k \leq m_{i} \leq k$, denote the margin of voters that party $i$ commands. Then there exists a population distribution in general position such that for any admissible design, party $i$ commands a margin of $m_{i}$ constituencies.

Proof. See appendix.

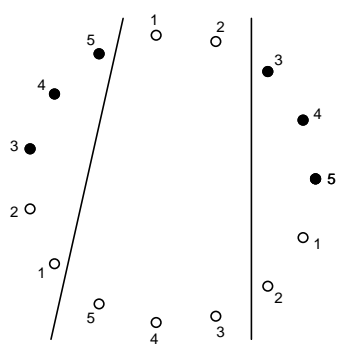

a: Black wins 2:1

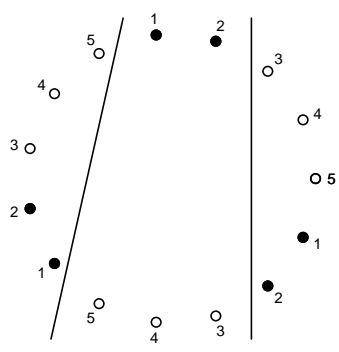

c: White wins 3:0

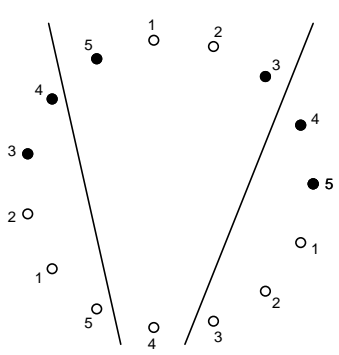

b: White wins 3:0

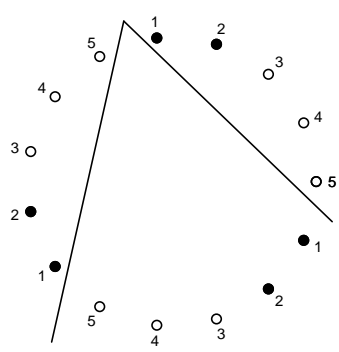

d: White wins 3:0

Figure 1 Polities with 6 black points, 9 white points, and 3 constituencies. For some configurations outcomes can range from 2:1 
majorities for Black (a) to 3:0 majorities for White (b). For others White wins by a majority of 3:0 for all admissible designs ((c) and (d)); in such cases there is no scope for gerrymandering. Note that all admissible constituencies include one point marked ' 1 ', one marked ' 2 ' and so on up to ' 5 '.

The proposition establishes that for some distributions convexity ensures that there is no scope for gerrymandering; in such cases the margins guaranteed by Proposition 1 can be returned but cannot be improved upon by gerrymanderers working for either side. The proof strategy of the Proposition exploits features of points arranged in convex position (that is, as vertices of a polygon). Consider a set of points arranged in this way and count them off into $k$ sequences $\left\{1,2, \ldots, \frac{n}{k}\right\}$ (see figure 1). Any admissible design must have in each constituency one point labeled ' 1 ', one point labeled ' 2 ' and so on up to $\frac{n}{k}$. As a result any distribution that allocates the first $\left(\frac{n}{k}-1\right) \frac{1}{2}$ indices to one group and the last $\left(\frac{n}{k}-1\right) \frac{1}{2}$ to the other group and assigns the remaining points to index $\left(\frac{n}{k}+1\right) \frac{1}{2}$ within each constituency achieves the desired distribution.

Figure 1 illustrates both propositions. We see that for a given division of votes, for some populations each group can, through gerrymandering, achieve up to $\min \left(\left\lfloor\frac{2 k}{n+k} v\right\rfloor, k\right)=\frac{v}{3}$. For other distributions the minimum provided in Proposition 1 is also a maximum.

\section{Conclusion}

Consistent with the argument of Vickrey (1961) we find that a shape constraint on constituency boundaries provides little insurance against gerrymandering. Whereas the classic image of a gerrymandered constituency involves tortured shapes, majority parties can generally ensure that vote leads translate into constituency leads even if they constrain themselves to regular shapes. This allows for very large distortions. In the UK for example with approximately 650 constituencies and 50 million voters, full control over constituency boundaries is sufficient to convert a one thousandth of a percent vote lead into $100 \%$ constituency control.

Do the results support the claim that compactness favors majority parties and more dispersed parties (Lowenstein and Steinberg, 1985)? The results do support the argument that a convexity constraint hurts minorities. Convexity does not prevent gerrymanderers supporting a 
party with a $k$ seat lead from controlling all constituencies, but it can prevent a minority party with a $k$ seat lag from gaining any (even if such a party could otherwise control up to $k-1$ seats). There are some benefits for minorities however: in close races (with margins between $-k$ and $k$ ) minorities can translate their vote margin into a seat margin and, for some distributions, they can be guaranteed that a majority gerrymanderer cannot force an even worse outcome upon them. However these benefits only arise for a very constrained set of contexts.

The results do not support the argument that compactness disadvantages more concentrated minority or majority groups confronted with gerrymandering by majority groups since, at least when operationalized as a convexity constraint, majority gerrymandering is unconstrained by distributions except for exceptionally close races. Whether compactness constrains gerrymanderers favoring concentrated minorities more than it does gerrymanderers favoring dispersed minorities remains an open question.

We close by emphasizing a final, deeper, implication of this analysis. At the outset we noted that convexity is a necessary but not a sufficient criterion for an optimal design under a wide range of compactness measures. Thus one might argue that the results of Proposition 1 may be avoided for designs that require more than convexity (such as the dispersion requirement in Fryer and Holden (2007)). This is correct. However the results of Proposition 2 are relevant for all criterion that include convexity: if all convex designs award all constituencies to one side then so do all designs that require more than convexity. Moreover, for such distributions, this is the case no matter which side controls the districting. This has important implications for normative assessments of gerrymandering. Perhaps the most prominent normative critique of gerrymandering is that it results in seat shares that poorly reflect vote shares (see for example the discussion in Gelman and King (1994)). Yet as is clear from Proposition 2, distributions exist such that any division into convex districts will produce such a mismatch. In such cases having "fairer" outcomes requires a gerrymandering that violates compactness. This clarifies the fact that the problem of mismatch can not simply be attributed to the gerrymander but is inherent in the use of single member districts in the mapping from votes to seats. 


\section{References}

Altman, M. (1998): "Modeling the effect of mandatory district compactness on partisan gerrymanders," Political Geography, 17, 989 1012 .

Bespamyatnikh, S., D. Kirkpatrick, and J. Snoeyink (2000): "Generalizing Ham Sandwich Cuts to Equitable Subdivisions," Discrete and Computational Geometry, 24, 605-622.

Chambers, C. P., And A. D. Miller (2009): "A Measure of Bizarreness," Working Paper, California Institute of Technology.

Friedman, J. N., ANd R. T. Holden (2008): "Optimal Gerrymandering: Sometimes Pack, But Never Crack," American Economic Review, 98(1), 113-144.

Fryer, R. G. J., AND R. T. Holden (2007): "Measuring the Compactness of Political Districting Plans," NBER Working Papers, 13456 .

Gelman, A., And G. King (1994): "Enhancing Democracy Through Legislative Redistricting," American Political Science Review, 88(3), 541-559.

Kaneko, A., M. Kano, And K. Suzuki (2004): "Path Coverings of Two Sets of Points in the Plane," in Towards a theory of geometric graphs, ed. by J. Pach, vol. 342, pp. 99-111. Contemporary Mathematics series of AMS.

Lowenstein, D. H., And J. Steinberg (1985): "The Quest for Legislative Districting in the Public Interest: Elusive or Illusory?," UCLA Law Review, 33, 1-75.

Niemi, R. G., B. Grofman, C. Carlucci, and T. Hofeller (1990): "Measuring Compactness and the Role of a Compactness Standard in a Test for Partisan and Racial Gerrymandering," The Journal of Politics, 52(4), 1155-1181.

Polsby, D. D., And R. D. Popper (1991): "The third criterion: Compactness as a procedural safeguard against partisan gerrymandering.," Yale Law and Policy Review, 9, 301-353. 
Stern, R. S. (1974): "Political gerrymandering: a statutory compactness standard as an antidote for judicial impotence.," The University of Chicago Law Review, 41, 398-416.

TAYLOR, P. J. (1973): "A new shape measure for evaluation electoral district patterns.," The American Political Science Review, 67, 947950 .

Vickrey, W. (1961): "On the prevention of gerrymandering," Political Science Quarterly, 76, 105-110.

Young, H. P. (1988): "Measuring the compactness of legislative districts," Legislative Studies Quarterly, 13, 105-115. 


\section{Appendix}

Proposition 2 Let $m_{i},-k \leq m_{i} \leq k$, denote the margin of voters that party $i$ commands. Then, there exists a population distribution in general position such that for any admissible design, party $i$ commands a margin of $m_{i}$ constituencies.

Proof. Let $P$ denote the set of points and $P_{i}$ and $P_{-i}$ the subsets belonging to group $i$ and its rival. Label the $n$ points $x_{0}$ through $x_{n-1}$ such that $j\left(\bmod \frac{n}{k}\right)<\frac{n+k}{2 k} \rightarrow x_{j} \in P_{i}$ and $j\left(\bmod \frac{n}{k}\right)>\frac{n+k}{2 k} \rightarrow x_{j} \in$ $P_{-i}$. Arrange them sequentially such that each point is a vertex of a convex polygon. Associate with each vertex a group $K_{h}$ according to $j$ $(\bmod k)=h \rightarrow x_{j} \in K_{h}$. To establish the proposition we demonstrate that for any admissible design, each cell contains exactly one point from each group $K_{h}$.

For any point $x_{j}$ in collection $A$, we say that $x_{(j+z)}(\bmod k)$ is 'next' to $x_{j}$ in $A$ if $z=\left\{\min _{s} \in\{1,2, \ldots, n-1\} \mid x_{j+s}(\bmod k) \in A\right\}$.

Now consider some admissible partition. We show that for any point $x_{g}$ in cell $C$, if $x_{g} \in K_{h}$ then the point next to $x_{g}$ in $C$ is in $K_{h+1}\left(\bmod \frac{n}{k}\right)$. Without loss of generality set $g=0$. Now assume to the contrary that $x_{w}$ is next to $x_{0}$ in $C$ but is not an element of $K_{1}$. Let $\bar{X}=\left\{x_{1}, x_{2}, \ldots, x_{w-1}\right\}$ and $\underline{X}=\left\{x_{w+1}, x_{w+2}, \ldots, x_{n}\right\}$ denote the collections of points between $x_{0}$ and $x_{w}$ (moving in different directions around the polygon). Since the points in $P$ are vertices on a convex polygon, the collection $\bar{X}$ lies on one side of the line that contains $x_{0}$ and $x_{w}$ and the collection $\underline{X}$ lies on the other. Since $x_{w}$ is next to $x_{0}$ in $C$ but is not an element of $K_{1}$ the cardinality of $\bar{X}$ is not evenly divisible by $k$ and so at least one point in $\bar{X}$ is in a cell together with some point in $\underline{X}$. Let $a$ and $b$ denote two such points. Since $P$ is in convex position we have that the line segment joining $a$ and $b$ intersects with the line segment joining $x_{0}$ and $x_{w}$. But then the (convex) cells containing $a$ and $b$ and $x_{0}$ and $x_{w}$ are not disjoint. This yields a contradiction, thus $x_{w}$ lies in $K_{1}$. By induction each point in $K_{0}$ shares a cell with a point in $K_{1}, K_{2}, \ldots, K_{\frac{n}{k}}$, the number of constituencies commanded by group $i$ corresponds exactly to the number of elements in collection $K_{\frac{n+k}{2 k}}$, and so $i$ 's margin in constituencies is equal to its margin in voters. 